\title{
All our knowledge begins with the antisenses
}

\author{
Ethan M. Goldberg ${ }^{1,2,3,4}$ \\ 'Division of Neurology, Department of Pediatrics and ${ }^{2}$ The Epilepsy NeuroGenetics Initiative, The Children's Hospital of Philadelphia, Philadelphia, Pennsylvania, USA. ${ }^{3}$ Department of Neuroscience and \\ ${ }^{4}$ Department of Neurology, The University of Pennsylvania Perelman School of Medicine, Philadelphia, Pennsylvania, USA.
}

\begin{abstract}
Epilepsy is the neurological disorder defined by spontaneous recurrent seizures, which are abnormal patterns of electrical discharge in the brain. A major advance in neurology over the last $\mathbf{2 0}$ years is the identification of genetic variation as an important cause of epilepsy, and in particular as a cause of the epileptic encephalopathies, defined by childhood-onset, treatment-resistant epilepsy accompanied by developmental delay leading to intellectual disability. Unfortunately, this progress in genetic diagnosis has yet to translate to effective precision or targeted therapeutics. However, in this issue of the $\mathrm{JCI}, \mathrm{Li}$ and Jancovski et al. use antisense oligonucleotides (ASO) to treat or prevent epilepsy and epilepsy-associated cognitive and behavioral comorbidities in a mouse model of SCN2A encephalopathy, paralogous to the recurrent human variant SCN2A c.5645G >A (p.R1882Q) associated with epileptic encephalopathy. These findings may inform the development of targeted or personalized therapies for what is currently an incurable and largely untreatable disorder.
\end{abstract}

\section{SCN2A-related neurodevelopmental disorders}

Over 100 monogenic causes of epilepsy have so far been identified, with an enrichment for genes encoding ion channels, neurotransmitter receptors, and synaptic molecules. Among these, each of the four brain-expressed $\mathrm{Na}^{+}$ion channels, SCN1A, $2 A, 3 A$, and $8 A$, are established epilepsy genes. De novo pathogenic variants in $S C N 2 A$ associate with a spectrum of neurodevelopmental disorders, including benign neonatal or infantile-onset epilepsy, autism spectrum disorder (ASD) and intellectual disability (ID), and early-onset epileptic encephalopathy (SCN2A encephalopathy) (1). Nav1.2-containing $\mathrm{Na}^{+}$channels are important for generating action potential in brain neurons and for regulating synaptic plasticity via back-propagation into neuronal dendrites $(2,3)$. Studies of $\mathrm{Na}^{+}$channels containing disease-associated Nav1.2 variants have led to the schema under which gain of function (GoF) due to missense variants in $S C N 2 A$ (e.g., with increased persistent current) associates with epileptic encephalopathy (4-6). In contrast, loss of function (LoF) due to missense variant or SCN2A deletion (with less $\mathrm{Na}^{+}$current), causes ASD/ID that is sometimes accompanied by typically mild epilepsy. Hence, one could envision a framework in which blocking Nav1.2-containing $\mathrm{Na}^{+}$channels or decreasing Nav1.2 expression could treat $S C N 2 A$ encephalopathy due to $\mathrm{GoF}$ variants. That seizures, in some cases, respond to $\mathrm{Na}^{+}$channel-blocking antiseizure medications $(7,8)$ provides support for this idea.

\section{Antisense oligonucleotides for epilepsy}

Enter antisense oligonucleotides (ASOs). ASOs are synthetic oligonucleotides (short strands of DNA or RNA) engineered to specifically bind to a unique target sequence (Figure 1). ASO drug candidates are designed to manipulate levels of an mRNA

\section{Delated Article: https://doi.org/10.1172/JCl152079}

Conflict of interest: The author has declared that no conflict of interest exists.

transcript that encodes a target protein of interest. For example, gapmer ASOs target mRNA for degradation by ribonuclease $\mathrm{H}(\mathrm{RNaseH})$. An alternative approach involves ASO binding of pre-mRNA to modulate splicing via steric hindrance (9).

Currently, there are more than 10 FDA-approved ASOs. One example includes nusinersen (marketed as Spinraza) for spinal muscular atrophy $(10,11)$, a pediatric neuromuscular disease due to reduced $S M N$ protein secondary to biallelic LoF variants in the SMN1 gene. Binding of nusinersen to an intronic splicing silencer between exons 7 and 8 of the SMN2 pre-mRNA facilitates exon 7 inclusion into the SMN2 mRNA and thereby increases levels of full-length, functional SMN protein in the central nervous system (Figure 1). There are more than 50 ASOs in various stages of clinical trials, with many others under preclinical development (12).

Stoke Therapeutics recently launched the phase 1/2a MONARCH study of STK001 for the treatment of Dravet syndrome (ClinicalTrials.gov, NCT04442295), an epileptic encephalopathy due to heterozygous LoF variants in SCN1A encoding the voltage-gated $\mathrm{Na}^{+}$channel subunit Nav1.1. STK-001 is an ASO designed to exploit the presence of an SCN1A poison exon (13, 14). The small, noncoding exon contains a within-frame STOP codon such that incorporation of this exon into the full-length transcript leads to premature truncation and nonproductive splicing that triggers nonsense-mediated decay. STK-001 binds to pre-mRNA to alter splicing and block inclusion of the poison exon into the SCN1A mRNA, the effect of which is to increase protein expression $(15,16)$. The authors refer to this method as targeted augmentation of nuclear gene output (TANGO). Intracerebroventricular (i.c.v.) injection of TANGO into developing P2 or P14 Scn1a ${ }^{+/-}$mouse pups led to reduced seizures and increased survival (15).

Previously, Lenk et al. used an ASO approach to target another voltage-gated $\mathrm{Na}^{+}$channel gene, $\mathrm{Scn} 8 a$, in a mouse model 
A

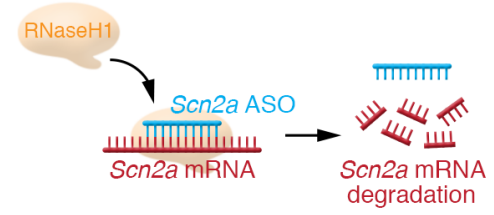

B

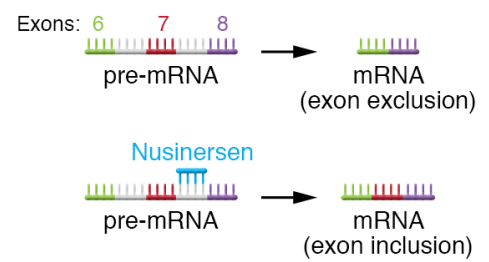

C

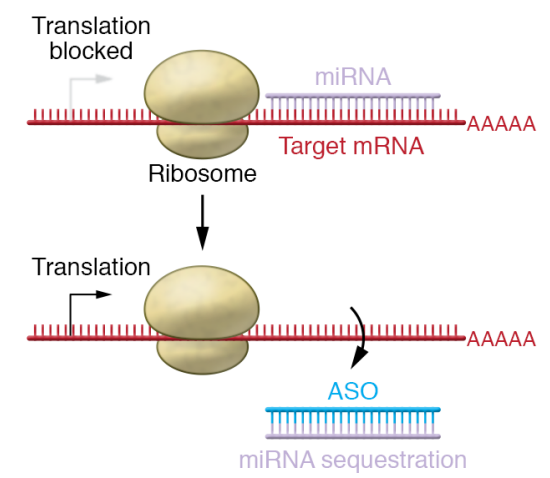

Figure 1. Antisense oligonucleotide strategies for therapeutic targeting of mRNA transcripts.

(A) Schematic showing the basic gapmer ASO approach taken by $\mathrm{Li}$ and Jancovski et al. (20) to target $\operatorname{Scn} 2 a$ in an experimental mouse model of SCN2A encephalopathy. (B) A splice-modifying ASO as in nusinersen (Spinraza), which alters the pre-mRNA splicing of SMN2 to facilitate integration of exon 7 into the mRNA to produce functional full-length protein. (C) miRNA-targeting ASO prevents miRNAs of interest from binding to a given RNA.

of SCN8A encephalopathy (17). Mice expressing the Scn8a-p.Arg1872Trp missense variant (18) are healthy prior to seizure onset, which occurs at P14-P16, then die within 24 hours. $\mathrm{Na}^{+}$channels containing Nav1.6-Arg1872Trp exhibit GoF. Hence, the authors generated an Scnsa ASO that decreased both WT and variant transcripts, but not other $\mathrm{Na}^{+}$channel transcripts. It is important to note that individuals who are heterozygous for LoF variants or lack $S C N 8 A$ due to deletion show mild intellectual disability with or without ataxia. Moreover, an Scn8a null mutation (lacking any functional Nav1.6) in mice is lethal (19); hence, marked reduction in Scn8a may prove detrimental (similar to the situation in Li and Jancovski et al. regarding Scn2a; ref. 20). Lenk et al. (17) attempted to titrate the ASO dose to partially reduce Scnsa mRNA and Nav1.6 protein while still maintaining sufficient levels to observe a therapeutic effect. Scn8a ASO delivered via i.c.v. injection at $\mathrm{P} 2$ prolonged survival and delayed epilepsy onset in Scn8a-p.Arg1872 Trp mice. Mice eventually exhibited epilepsy and death; however, repeated dosing prolonged survival (17).

\section{ASO for SCN2A encephalopathy}

In this issue of the JCI, Li and Jancovski et al. (20) used a gapmer ASO targeting Scn $2 a$ mRNA to treat or prevent epilepsy and epilepsy-associated cognitive and behavioral comorbidities in a mouse model of SCN2A encephalopathy. Scn2ap.R1883Q mice (hereafter, Q/+ mice) are homologous to the recurrent human variant SCN2A c.5645G>A (p.R1882Q) associated with epileptic encephalopathy (1). This variant produced $\mathrm{Na}^{+}$currents in heterologous cell systems with increased persistent $\mathrm{Na}^{+}$current and, hence, likely GoF. Q/+ mice exhibited a severe, earlyonset seizure phenotype, with spontaneous tonic seizures seen as early as P1, death as early as P13, and median survival time of 18 days. All mice were deceased by P30. Since the authors could not propagate mice with this phenotype, they cleverly generated an experimental animal model in which a targeting vector was delivered to mouse embryonic stem cells to yield $100 \%$ ES cell-derived Q/+ founder mice after implantation into and delivery by WT surrogate host dams.

The authors confirmed that i.c.v. injection of the Scn2a ASO successfully downregulated Scn $2 a$ mRNA and Nav1.2 protein (both variant and WT) with no off-target effect on other voltage-gated $\mathrm{Na}^{+}$channels. An effective dose was determined for $50 \%$ reduction $\left(\mathrm{ED}_{50}\right)$ of $\operatorname{Scn} 2 a$ mRNA levels at different ages. A single i.c.v. injection of $\mathrm{ED}_{50} S c n 2 a$ ASO into $\mathrm{P} 1 \mathrm{Q} /+$ mice prolonged median survival to $\mathrm{P} 47$ with approximately $10 \%$ of mice surviving to the experimental endpoint at P80, compared with control ASO. Scn2a ASO almost completely eliminated seizures as determined via continuous video EEG at various time points. Repeated administration of $\operatorname{Scn} 2 a$ ASO further extended survival. Scn $2 a$ ASO $\mathrm{ED}_{50}$ i.c.v. injection at P14-P16 also extended lifespan, suggesting that treatment could be effective if initiated after symptom onset. Treated $\mathrm{Q} /+$ mice underwent a battery of behavioral and cognitive testing and minimal abnormalities were identified. However, Scn2a ASO $\mathrm{ED}_{80}$ did result in impaired motor function as well as behavioral changes relative to untreated WT mice (comparison to untreated $\mathrm{Q} /+$ mice was impossible as all mice were deceased at the relevant time points).

$\mathrm{ED}_{50}$ i.c.v. injection into WT mice at P1 did not affect body weight or survival, but $\mathrm{ED}_{80}$ injection led to decreased body weight and early mortality, consistent with early lethality observed in $\operatorname{Scn} 2 a^{-/-}$mice. WT mice injected with $\mathrm{ED}_{50}$ did spend less time in the closed arm of an elevated plus maze and demonstrated impairments on a grid walk assay.

These results show that $S c n 2 a$ ASO can treat or prevent epilepsy and epilepsyassociated comorbidities, and prolong survival in an experimental mouse model of SCN2A encephalopathy with minimal apparent side effects if closely titrated, which could be achieved in the preclinical system but may be more difficult in humans.

Such results are impressive. However, one question that was not fully addressed by $\mathrm{Li}$ and Jancovski et al. is the exact mechanism whereby Scn2a ASO exerts this effect at the neuronal and network levels, and if the observed effects were actually due to downregulation of variant Scn $2 a$. The authors provided some data demonstrating relatively subtle increases in the excitability of pyramidal neurons in layer $2 / 3$ primary somatosensory neocortex in acute brain slices prepared from P10-P12 Q/+ mice. Neuronal excitability was accompanied by or due to increased input resistance and a lower rheobase (the minimal current injection required to generate an action potential). It seems unlikely that the profound phenotype in the $\mathrm{Q} /+$ mice was due to this particular electrophysiological abnormality. Demonstration of cellular and circuit abnormalities would provide further support for the biological premise.

An important control or complementary experiment would be to treat another mouse epilepsy model with Scn2a ASO, such as a model of acquired epilepsy. Perhaps Scn $2 a$ ASO exerts a nonspecific antiseizure effect? As noted above, many 
antiseizure medications act via $\mathrm{Na}^{+}$channel blockade, and $\mathrm{Na}^{+}$channel-blocking agents show efficacy in treating seizures in some cases of SCN2A encephalopathy. Perhaps the Scn $2 a$ ASO acts nonspecifically as a $\mathrm{Na}^{+}$channel-blocking antiseizure medication. An allele-specific ASO (targeting only the variant allele) could address this question and could potentially achieve the same or greater effect with fewer off-target effects, yet would have even more narrow clinical applicability.

ASO delivered intrathecally can target the peripheral nervous system in humans as demonstrated by the success of Spinraza. But in the examples described above for TANGO in Scn1a ${ }^{+/-}$mice, Scnsa ASO for $S c n 8 a$ encephalopathy, and $\operatorname{Scn} 2 a$ ASO for $\operatorname{Scn} 2 a$ encephalopathy in $\mathrm{Li}$ and Jancovski et al., the compound was delivered via i.c.v. Li and Jancovski et al. show widespread distribution of ASO in mouse brain that is not likely achievable in humans via intrathecal injection as ASOs cross the blood-brain barrier inefficiently. It would be useful to know the extent of distribution needed in mouse brain to achieve the therapeutic effects observed by Li and Jancovski et al.

\section{Considering safety and efficacy}

An initial question for the future is whether ASO therapy is safe, and it largely appears to be. However, for some genes, including for epilepsy-associated ion channel genes, both $\mathrm{GoF}$ and LoF are pathogenic. In the case of $S C N 2 A$, LoF is an important cause of ASD/ID. Could an SCN2A ASO convert patients from an epileptic encephalopathy to an ASD/ID phenotype? Allele-specific ASO could potentially avoid this issue.

It is also known that plastic changes occur in brain circuits in response to pathogenic variants of disease-associated genes. Two recent papers show that the electrical excitability of Nav1.2-expressing excitatory neurons in striatum and neocortex is actually enhanced, rather than impaired, in mice lacking $\operatorname{Scn} 2 a(21,22)$, due to complex interactions between and compensatory reorganization of $\mathrm{Na}^{+}$and potassium $\left(\mathrm{K}^{+}\right)$currents. What changes might occur during development in Scn $2 a$ encephalopathy remains unknown. However, the safety and efficacy of a given ASO could depend on the timing of delivery during development. It is possible that there is a critical period within which knockdown of $S C N 2 A$ might determine a given clinical outcome. This therapeutic window may make early and rapid genetic diagnosis in the epilepsies more critical.

\section{Considering equity}

Finally, an important question for the future is the potential scope across which ASO therapies can eventually be made available given that there are over 700 genetic causes of intellectual and developmental disability. The San Diego, California, USA-based nonprofit company n-Lorem, working with Ionis Pharmaceuticals (which contributed to the $\mathrm{Li}$ and Jancovski et al. paper), aims to develop ASO-based therapies for children with ultrarare diseases affecting one or a small handful of patients, and offer such therapies for free (free, at least, to the patient). It remains to be seen how the field can and should best allocate resources and address issues related to equity of access to this promising therapy.

ASOs potentially open an exciting avenue toward the treatment of rare neurological diseases that might previously have been considered unapproachable via other therapies. Questions remain related to equity of access: how should targets be prioritized for therapy development, and based on what criteria? Disease severity, lack of available alternative therapies such as candidate small molecules, existence of robust natural history data, knowledge of the ASO target and disease pathomechanisms, and total number of patients who might stand to benefit, could factor into such calculations.

Nevertheless, early studies applying ASO technology to the treatment of epileptic encephalopathy and other neurodevelopmental disorders, including the study by $\mathrm{Li}$ and Jancovski et al., provide hope for a better tomorrow for patients with epilepsy and their families.

\section{Acknowledgments}

Work in the author's laboratory is supported by the Dravet Syndrome Foundation, March of Dimes, Burroughs Wellcome Fund, and National Institutes of Health grants K08 NS097633, R01 NS110869, and R01 NS119977. The author would like to thank Eric D. Marsh for comments on the manuscript and Melody Cheng for assistance with generation of the figure.
Address correspondence to: Ethan M. Goldberg, The Children's Hospital of Philadelphia, Abramson Research Center Room 502A, 3615 Civic Center Boulevard, Philadelphia, Pennsylvania 19104, USA. Phone: 215.590.6894; Email: goldberge@ chop.edu.

1. Sanders SJ, et al. Progress in understanding and treating SCN2A-mediated disorders. Trends Neurosci. 2018;41(7):442-456.

2. $\mathrm{Hu} \mathrm{W}$, et al. Distinct contributions of $\mathrm{Na}(\mathrm{v}) 1.6$ and $\mathrm{Na}(\mathrm{v}) 1.2$ in action potential initiation and backpropagation. Nat Neurosci. 2009;12(8):9961002.

3. Spratt PWE, et al. The autism-associated gene Scn2a contributes to dendritic excitability and synaptic function in the prefrontal cortex. Neuron. 2019;103(4):673-685.

4. Scalmani $\mathrm{P}$, et al. Effects in neocortical neurons of mutations of the $\mathrm{Na}(\mathrm{v}) 1.2 \mathrm{Na}+$ channel causing benign familial neonatal-infantile seizures. J Neurosci. 2006;26(40):10100-10109.

5. Wolff M, et al. Genetic and phenotypic heterogeneity suggest therapeutic implications in SCN2A-related disorders. Brain. 2017;140(5):1316-1336

6. Ben-Shalom R, et al. Opposing effects on NaV1.2 function underlie differences between SCN2A variants observed in individuals with autism spectrum disorder or infantile seizures. Biol Psychiatry. 2017;82(3):224-232.

7. Dilena R, et al. Efficacy of sodium channel blockers in SCN2A early infantile epileptic encephalopathy. Brain Dev. 2017;39(4):345-348.

8. Foster LA, et al. Infantile epileptic encephalopathy associated with SCN2A mutation responsive to oral mexiletine. Pediatr Neurol. 2017;66:108-111.

9. Bennett CF. Therapeutic antisense oligonucleotides are coming of age. Annu Rev Med. 2019;70:307-321.

10. Corey DR. Nusinersen, an antisense oligonucleotide drug for spinal muscular atrophy. Nat Neurosci. 2017;20(4):497-499.

11. Finkel RS, et al. Treatment of infantile-onset spinal muscular atrophy with nusinersen: a phase 2, open-label, dose-escalation study. Lancet. 2016;388(10063):3017-3026.

12. Sztainberg Y, et al. Reversal of phenotypes in MECP2 duplication mice using genetic rescue or antisense oligonucleotides. Nature. 2015;528(7580):123-126.

13. Carvill GL, et al. Aberrant inclusion of a poison exon causes dravet syndrome and related SCN1A-associated genetic epilepsies. Am J Hum Genet. 2018;103(6):1022-1029.

14. Aziz MC, et al. Targeting poison exons to treat developmental and epileptic encephalopathy. Dev Neurosci. 2021;43(3-4):241-246.

15. Han Z, et al. Antisense oligonucleotides increase Scnla expression and reduce seizures and SUDEP incidence in a mouse model of Dravet syndrome. Sci Transl Med. 2020;12(558):eaaz6100.

16. Lim KH, et al. Antisense oligonucleotide modulation of non-productive alternative splicing upregulates gene expression. Nat Commun. 
2020;11(1):3501.

17. Lenk GM, et al. Scn8a antisense oligonucleotide is protective in mouse models of SCN8A encephalopathy and dravet syndrome. Ann Neurol. 2020;87(3):339-346.

18. Bunton-Stasyshyn RKA, et al. Prominent role of forebrain excitatory neurons in SCN8A encephalopathy. Brain. 2019;142(2):362-375.
19. Burgess DL, et al. Mutation of a new sodium channel gene, Scn8a, in the mouse mutant 'motor endplate disease'. Nat Genet. 1995;10(4):461-465.

20. Li M, et al. Antisense oligonucleotide therapy reduces seizures and extends life span in an SCN2A gain-of-function epilepsy model. JClin Invest. 2021;131(23):e152079.
21. Spratt PWE, et al. Paradoxical hyperexcitability from $\mathrm{Na}_{\mathrm{v}} 1.2$ sodium channel loss in neocortical pyramidal cells. Cell Rep. 2021;36(5):109483.

22. Zhang J, et al. Severe deficiency of the voltagegated sodium channel $\mathrm{Na}_{\mathrm{v}} 1.2$ elevates neuronal excitability in adult mice. Cell Rep. 2021;36(5):109495. 